\title{
Relevance of Metric-Free Interactions in Flocking Phenomena
}

\author{
Francesco Ginelli ${ }^{1,2}$ and Hugues Chaté ${ }^{1}$ \\ ${ }^{1}$ Service de Physique de l'État Condensé, CEA-Saclay, 91191 Gif-sur-Yvette, France \\ ${ }^{2}$ Institut des Systèmes Complexes de Paris Ille-de-France, 57-59 rue Lhomond, 75005 Paris, France
}

(Dated: October 29, 2018)

\begin{abstract}
We show that the collective properties of self-propelled particles aligning with their "topological" (Voronoi) neighbors are qualitatively different from those of usual models where metric interaction ranges are used. This relevance of metric-free interactions, shown in a minimal setting, indicate that realistic models for the cohesive motion of cells, bird flocks, and fish schools may have to incorporate them, as suggested by recent observations.
\end{abstract}

PACS numbers: 87.10.-e, 05.70.Fh, 64.60.-i

Active matter, where energy is spent locally to produce motion, such as in the cytoskeleton of living cells, displays a number of fascinating phenomena and raises important new theoretical questions [1]. One such general problem is that of the emergence of collective motion in large groups of self-propelled objects. Alongside the more traditional studies of animal behavior, the physics community focused, not surprisingly, on minimal models and their universal properties, following the seminal work of Vicsek et al. [2]. In many of these works, models consist of interacting particles and rely on metric thresholds and cut-offs to delimit various interaction zones [3].

Recent facts, however, point towards a metric-free, topological determination of the "neighbors" with whom a given individual interacts. The StarFlag project provided snapshots of reconstructed three-dimensional flocks of starlings of up to a few thousand birds. Statistical analysis revealed that a typical starling significantly interacts with its 7 or 8 closest neighbors, located at rather well-defined angular positions, but irrespective of their actual distance [4]. The authors then concluded to a "topological" determination of neighbors, although their results, obtained on flocks with a rather homogeneous density, can also be explained by a mere global rescaling of distances by each flock's density. Another hint at the relevance of metric-free interactions can be found in the recent work on crowd dynamics by Moussaïd et al. [5] who argue that pedestrians make decisions based on the "angular landscape" formed by surrounding others, who may screen out otherwise close neighbors. Finally, such a screening effect is probably important in (moving) cohesive groups of cells with direct cell-to-cell interactions.

In this Letter, we investigate the influence of metricfree, "topological" determination of neighbors on collective properties of self-propelled particles in a minimal setting. We introduce a "topological Vicsek model" in which constant-speed particles align their velocity with that of others forming their first Voronoi shell. We show that the introduction of Voronoi neighbors changes qualitatively the collective properties of the original model. At finite density, true long-range order arises but, contrary to the metric model, no density segregation is present and the transition to collective motion is continuous with non mean-field critical exponents that we determine numerically in two dimensions. Large flocks evolving in an infinite domain move and spread diffusively, but quasilong-range order nevertheless arises.

In the original, "metric", Vicsek model point particles align locally, in competition with some noise. The interaction consists in taking the (polar, ferromagnetic) average of velocities over all particles within unit distance. Two types of noise have been studied: the "angular" noise consists in adding a random angle to the orientation of the averaged velocity; with the "vectorial" noise, one adds a random vector to the average velocity before taking the orientation of the resulting vector to stream particles (for details, see, e.g. 7]). In all cases, particles perform uncorrelated random walks at strong noise, while they align perfectly in the absence of noise. An order-disorder transition happens in between, which was originally found continuous by Vicsek et al. [2]. If the existence, even in two dimensions, of a fluctuating collective motion phase with long-range order and algebraic correlations was established early by $\mathrm{Tu}$ and Toner [6], later studies [7 9] revealed that it is subdivided in two regions and that the transition is discontinuous due to the spontaneous emergence of dense, ordered, traveling solitary bands or sheets. The importance of these structures has been recognized implicitly by a number of groups [10] which tried to frustrate their emergence because they question the discontinuous character of the transition in the angular noise case. In the following, we restrict ourselves to the case of vectorial noise for which the discontinuous nature of the transition is admitted.

The topological Vicsek model with vectorial noise is defined like its metric counterpart: $N$ point particles move off-lattice at constant speed $v_{0}$. In two dimensions (to which we restrict ourselves below), particle $j$ is defined by its (complex) position $\mathbf{r}_{j}^{t}$ and orientation $\theta_{j}^{t}$, updated 
at unit time steps according to

$$
\begin{aligned}
& \theta_{j}^{t+1}=\arg \left[\sum_{k \sim j} \exp i \theta_{k}^{t}+\eta \mathcal{N}_{j}^{t} \xi_{j}^{t}\right] \\
& \mathbf{r}_{j}^{t+1}=\mathbf{r}_{j}^{t}+v_{0} \exp i \theta_{k}^{t+1},
\end{aligned}
$$

where $\xi$ is a random unit vector and $\eta$ the noise strength.

The key difference with the metric case is that the neighbors $k$ are chosen to be the $\mathcal{N}_{j}^{t}$ particles forming the first shell around particle $j$ in the Voronoi tesselation constructed from the particle positions at time $t$. With respect to its metric counterpart, this model has one less parameter since there is no interaction range. In most of the following, we used square domains of linear size $L$ with periodic boundary conditions. Without loss of generality, we rescale lengthscales in order to fix the density $\rho=N / L^{2}=1$. We are then left with two parameters, the microscopic speed $v_{0}$, which we keep constant at $v_{0}=0.5<1 / \sqrt{\rho}=1$, and our main control parameter, the noise strength $\eta$.

At large $\eta$, noise dominates the interaction, and one observes essentially random configurations. Decreasing the noise strength, the order parameter $\varphi(t)=\left|\left\langle\exp i \theta_{j}^{t}\right\rangle_{j}\right|$ increases to order one values (Fig. 1a). For a fixed noise strength in the ordered side, $\varphi$ decreases slightly with $L$, but slower than a power-law (Fig. 1b): our topological model, like the original Vicsek model, gives rise to true long-range polar order. Its ordered phase, though, is qualitatively different from that of the metric model: here, there is no coupling between local density and local order (Fig. 18), because neighbors in a sparse region are never disconnected, and thus low density does not necessarily induces disorder. The segregation mechanism which allows eventually for the emergence of bands in the metric model is absent. Indeed, scanning sizes up to $L=1024$, no band-like structure was detected.

Giant number fluctuations - a signature of fluctuating ordered active phases [11] - are nevertheless present, as in metric models: the variance $\Delta n$ of the number of particles present in square boxes of linear size $\ell$ scales like $n^{\alpha}$ with $\alpha \simeq 1.75>1$ where $n=\rho \ell^{2}$ is the average number of particles in the box (Fig. 1 1 ). This value of $\alpha$ is, however, significantly different from that known for the metric polar models, $\alpha=\frac{8}{5}$ [7, 14]. The exact origin of this discrepancy, beyond the above remark about the absence of a basic segregation mechanism, remains unclear: a good reason would be the occurence of longrange connections, allowed by the topological determination of neighbors, but this is not the case: distances to (Voronoi) neighbors are exponentially distributed in the ordered phase, which is qualitatively different from the Gaussian tail observed in the disordered phase, but both yield a small typical interaction range (Fig. 1f).

While they are no proof, the above findings, and in particular the absence of bands, hint that the transi- (a)

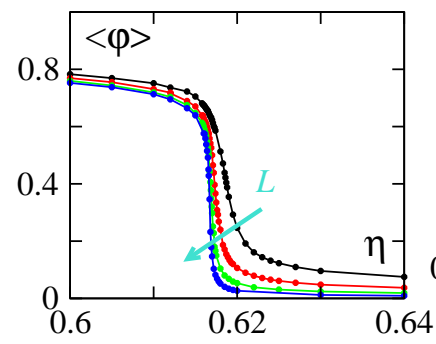

(c)

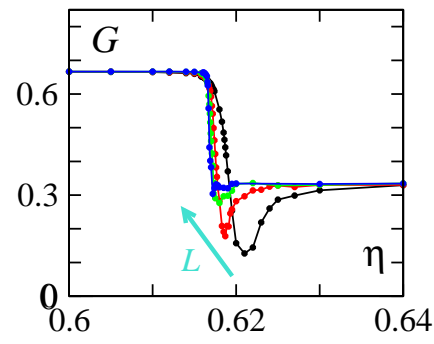

(e)
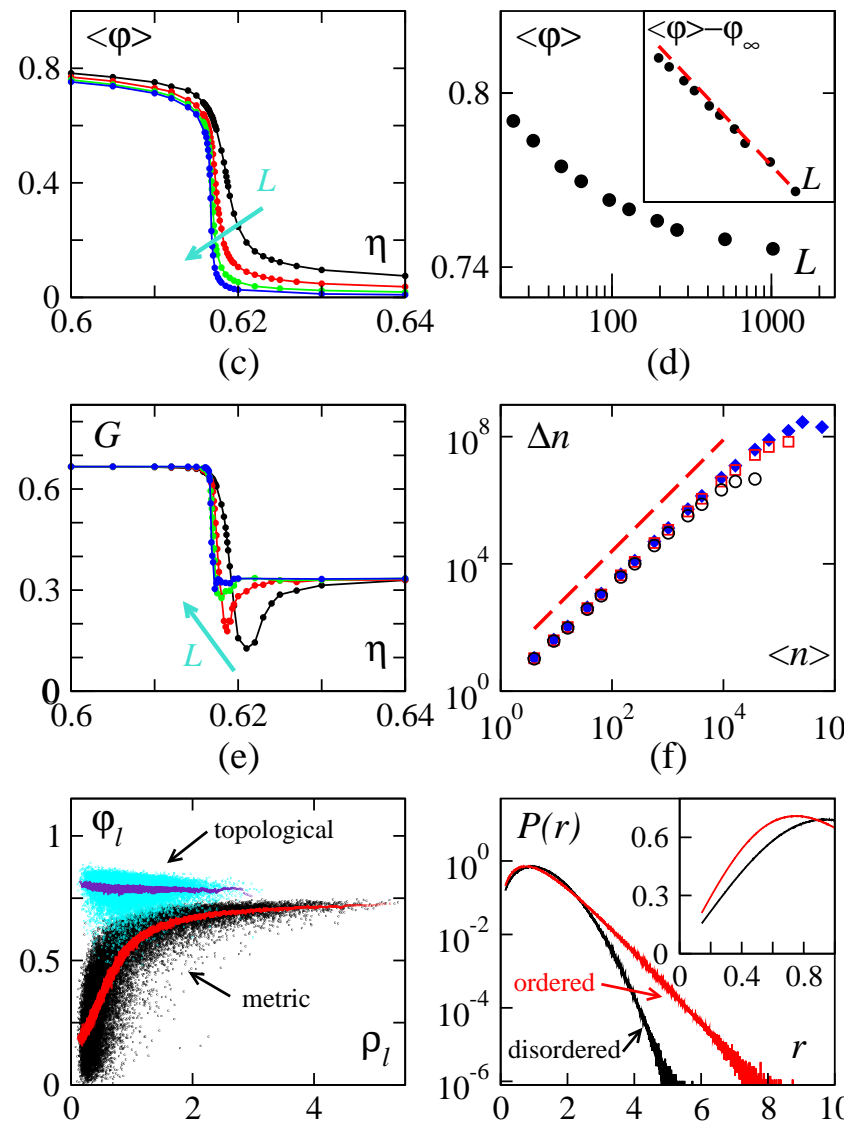

(d)

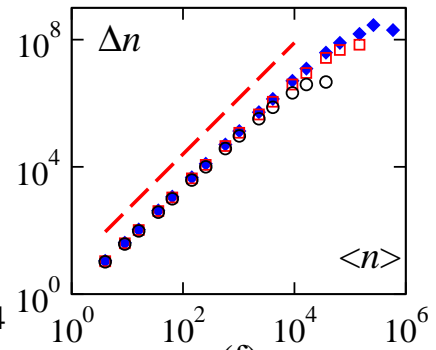

(f)

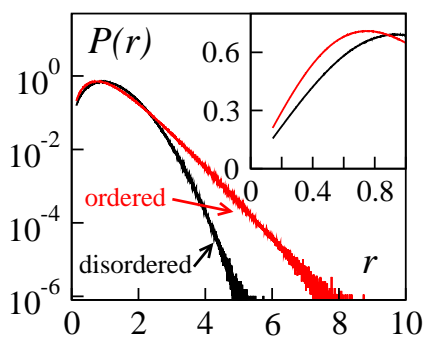

FIG. 1: (color online) (a) time-averaged order parameter $\langle\varphi\rangle$ vs $\eta$ for different system sizes $L$. From top to bottom (the cyan arrow points from smaller to larger systes sizes): $L=32$, 64, 128, 256. (b) $\langle\varphi\rangle$ vs $L$ at $\eta=0.6$ (logarithmic scales). Inset: same data from which $\varphi_{\infty}=0.741166$ has been subtracted. The dashed line marks a power law decay as $L^{-2 / 3}$. (c) Binder cumulant $G$ vs noise amplitude $\eta$ for different system sizes $L$ (same symbols and colors as in (a)). (d) Giant density fluctuations in the ordered phase. $\Delta n$ vs $n$ at $\eta=0.6$ for different system sizes: $L=256$ (circles), 512 (squares), 1024 (blue). The dashed red line has a slope 1.75. (e) Scatter plot of local order parameter $\varphi_{l}$ versus local density $\rho_{l}$ computed in boxes of size $\ell=16$ in the ordered phase. Black dots: Vicsek metric model with interaction distance $r_{0}=1$ and parameters $v_{0}=0.5, \eta=0.6, \rho=2$ and $L=256$. Cyan dots: topological model with $v_{0}=0.5, \eta=0.6, \rho=1$ and $L=512$. The lines passing through the middle of these two clouds of points are local averages in a moving window in $\rho_{l}$. (f) (normalized) distribution $P(r)$ of distances to Voronoi neighbours ( $L=128$ ). Black line: $\eta=0.8$ (disordered); red line $\eta=0.55$ (ordered). Both cases yield typical distances of order $r=1$. Inset: same in linear scales, showing that $P(r) \sim r$ as $r \rightarrow 0$.

tion of our topological model might not have the discontinuous character observed in its metric counterpart. The behavior of the so-called Binder cumulant $G=1-$ $\left\langle\varphi^{4}\right\rangle_{t} /\left(3\left\langle\varphi^{2}\right\rangle_{t}^{2}\right)$ confirms this observation: when decreasing the noise strength $\eta, G$ exhibits a dip towards val- 
(a)

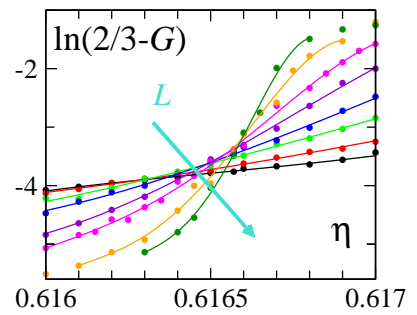

(c)

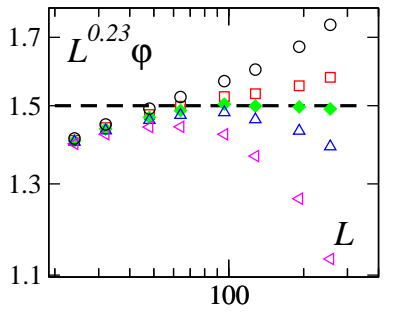

(b)

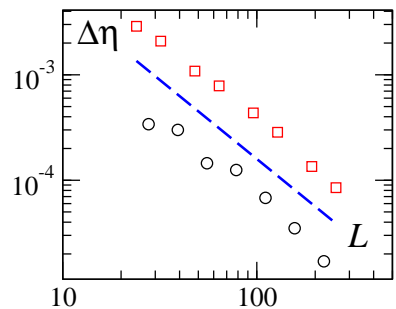

(d)

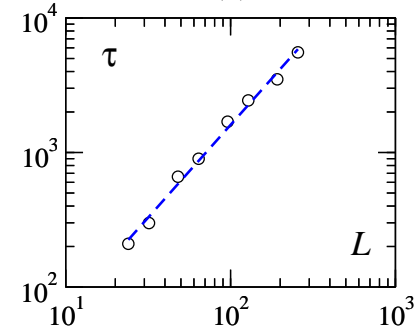

FIG. 2: (color online) (a) Zoom on the crossing area of the $G(\eta)$ curves obtained at different $L$ values $(L=$ $24,32,48,64,96,128,192,256)$. (The variable $\log \left(\frac{2}{3}-G\right)$ was chosen to produce the rather good quality polynomial fits shown with solid lines). (b) $\Delta \eta=\left|\eta_{\mathrm{c}}(L)-\eta_{c}^{\infty}\right|$ vs. $L$ for $\eta_{c}^{\infty}=0.61661$. Circles: $\eta_{\mathrm{c}}\left(L_{12}\right)$ is the crossing point between the $G(\eta)$ curves of panel (a) for two consecutive sizes $L_{1}$ and $L_{2}$. Squares: $\eta(L)$ is given by the position of the maximum of the susceptibility. The dashed line marks a power law decay as $L^{-1.5}$. (c) $\varphi \times L^{0.23}$ vs $L$ for different $\eta$ values using fitted order parameter curves (from top to bottom $\eta=0.6165$, $0.61658,0.61661,0.61664,0.6171)$. The flat dashed black line is a guide for the eye. (d) Autocorrelation time vs. $L$ at the critical point. The dashed line has a slope of 1.38 .

ues smaller than $\frac{1}{3}$ (the expected value in the disordered phase) before reaching $\frac{2}{3}$, the ordered value (Fig. 1:). If this dip were to sharpen and deepen to negative values when increasing system sizes, it would be the signature of a discontinuous transition [12]. But here, on the contrary, it gradually disappears as $L$ is increased (Fig. 11), definitely ruling out this possibility: in the infinite-size limit, the Binder cumulant varies monotonically through the transition, which is thus continuous. To estimate the values of the associated critical exponents, we performed a classic finite-size-scaling study [13], measuring the first moments of the order parameter as functions of $\eta$ for various systems sizes. The correlation time $\tau(\eta, L)$, estimated from the exponential decay of the autocorrelation function of the order parameter, was measured systematically and we made sure that our runs were always longer than $10^{3} \tau$. To locate the critical point, we determined the $\eta$ values at which the $G(\eta)$ curves obtained for different system sizes $L$ cross each other (Fig. 2a). Without corrections to scaling, these curves should cross at the asymptotic critical point $\eta_{\mathrm{c}}^{\infty}$ where the Binder cumulant takes a universal value $G_{\mathrm{c}}^{\infty}$. Here, the crossing points $\eta_{\mathrm{c}}\left(L_{1}, L_{2}\right)$ estimated for two consecutive sizes $L_{1}$ and $L_{2}$

TABLE I: Critical exponent and Binder cumulant values.

\begin{tabular}{cccccc}
\hline \hline $1 / \nu$ & $\beta / \nu$ & $\gamma / \nu$ & $\mathrm{z}$ & $\omega$ & $G_{\mathrm{c}}^{\infty}$ \\
\hline $1.5(1)$ & $0.23(3)$ & $1.49(5)$ & $1.38(5)$ & $0.67(20)$ & $0.615(2)$ \\
\hline \hline
\end{tabular}

are not identical but quickly converge, as $L_{12} \equiv \sqrt{L_{1} L_{2}}$ increases, to $\eta_{\mathrm{c}}^{\infty}=0.6166(1)$. If due to "ordinary" corrections to scaling, the convergence of the crossing points should be described by $\eta_{\mathrm{c}}\left(L_{1}, L_{2}\right)=\eta_{\mathrm{c}}^{\infty}+a L_{12}^{1 / \nu}$ and that of $G_{\mathrm{c}}\left(L_{1}, L_{2}\right)$ should be $G_{\mathrm{c}}\left(L_{1}, L_{2}\right)=G_{\mathrm{c}}^{\infty}+b L^{-\omega}$ where $\omega$ is the first correction to scaling exponent [13]. These scaling laws are well verified by our data using the above estimate of $\eta_{\mathrm{c}}^{\infty}$ (Fig. 2b, not shown for $\omega$ ). Similarly, the location of the maximum of the susceptibility $\chi=L^{d}\left(\left\langle\varphi^{2}\right\rangle_{t}-\langle\varphi\rangle_{t}^{2}\right)$ converges to $\eta_{\mathrm{c}}^{\infty}$ with the exponent $1 / \nu$, but here from above whereas it is from below for the effective thresholds $\eta_{\mathrm{c}}\left(L_{1}, L_{2}\right)$ determined using the crossings of the Binder cumulant curves. Using these two approaches to $\eta_{\mathrm{c}}^{\infty}$ allows us to narrow our estimate to $\eta_{\mathrm{c}}^{\infty}=0.61661(3)$ and yields $1 / \nu=1.5(1)$ (Fig. 2b). Next, we verified that our finite-size estimates of the order parameter at the critical point satisfy $\varphi\left(L, \eta_{\mathrm{c}}^{\infty}\right) \propto L^{-\beta / \nu}$ (Fig. 2r), yielding $\beta / \nu=0.23(3)$. Making use of the usual so-called hyperscaling relation $2 \beta-\gamma=\nu d$ where $d$ is the space dimension, the above results allow us to complete our estimates of the static exponents yielding $\gamma / \nu=1.54(6)$. Additional consistency checks of the above numbers (using various other quantities) were performed. For instance, the scaling of the maximum of the susceptibility $\chi_{\max }(L) \propto L^{\gamma / \nu}$ is rather nicely verified, yielding an estimate of $\gamma / \nu=1.49(5)$ compatible with the above numbers (not shown). Finally, the divergence with $L$ of the correlation time estimated at criticality, $\tau\left(\eta_{c}^{\infty}, L\right) \propto L^{z}$, yields an estimate of the dynamical exponent $z=1.38(5)$ (Fig. 2d). A summary of our estimates is in Table I. They indicate that the critical properties of our model do not correspond to mean-field values (where, for instance $\beta=\frac{1}{2}$ ), nor to any known universality class.

The transition to collective motion in the Vicsek topological model and its ordered phase at finite density are thus different from that of its metric counterpart. Our model also possesses non-trivial properties in the zerodensity limit of a large group of $N$ particles evolving in an infinite domain. Even though no attractive interaction is present, subgroups can never detach to become independent, because of the topological determination of neighbors. This insures a weak cohesion: a finite group typically expands diffusively at large times, but is nevertheless able to order at low enough noise strengths (Fig. 3a), something obviously impossible in metric models without attractive interactions. Remarkably, the order parameter remains statistically stationary, even on very long time scales, in spite of the constant expansion of the flock. The center of mass of the particles performs a persistent 
(a)

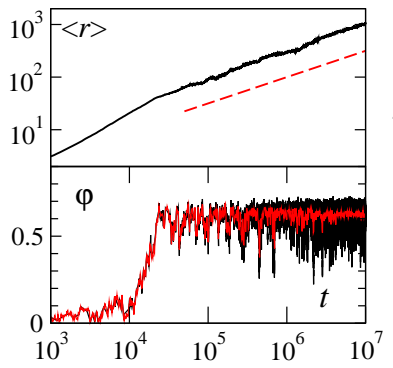

(c)

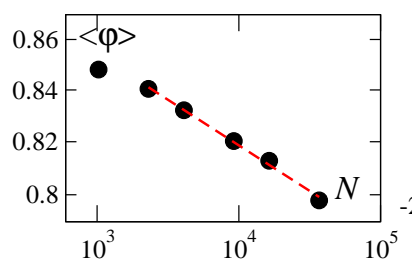

(b)
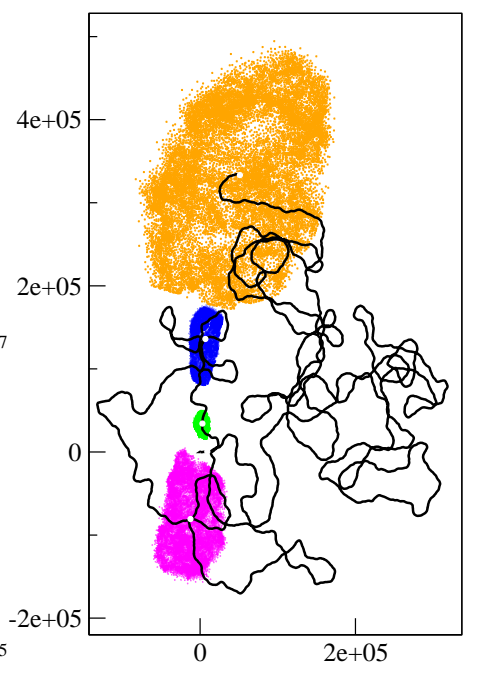

FIG. 3: (color online) Ordered flock in an infinite domain (initial conditions: random positions and random orientations in a unit circle). (a) mean distance $\langle r\rangle$ between Voronoi neighbors (top) and $\varphi(t)$ (bottom) vs. time $\left(N=2^{14}, \eta=0.61\right)$ : after transient, the flock is ordered and spreads diffusively $(\langle r\rangle \sim \sqrt{t})$, indicated by the dashed line). The red data in the bottom panel is a moving average on a window of fixed length in $\ln (t)$. (b) Trajectory of the center of mass for $t \in\left[1,10^{7}\right]$ plus snapshots of the flock in the asymptotic regime at $t=32 \times 10^{3}$ (red), $128 \times 10^{3}$ (green), $512 \times 10^{3}$ (blue) $2048 \times 10^{3}$ (magenta) and $10^{7}$ (orange) (same run as in (a)). The trajectory shows no sign of "aging" as the flock expands diffusively. (c) $\langle\varphi\rangle$ vs $N$ in logarithmic scales $(\eta=0.5$, time averages over $10^{7}$ timesteps after discarding an transient of $10^{6}$ ). Dashed line: power law with exponent -0.074 .

random walk whose turns mostly reflect the fluctuations of the order parameter and are not accompanied by largescale changes in the group structure or shape (Fig. $3 \mathrm{~b}$ ). In other words, the orientation axes of the particles turn, but there is hardly any rotation of mass, just marginal changes in the shape and the Voronoi tesselation.

Thus, if space is rescaled by a dynamical length reflecting the expansion of the group $(\ell \sim \sqrt{t})$, our system is mapped onto one where the microscopic velocity now goes to zero like $1 / \sqrt{t}$, evolving on a (quasi-) static Voronoi tessellation network: up to the rare rearrangements mentioned above, this is an XY model. Indeed, as for the two-dimensional XY model, only quasi-longrange order arises in the zero-density limit of our topological model: Increasing $N$, the average order parameter decreases slightly, as in the finite-density case, but this time goes to zero as a power-law (Fig. 35).

To summarize, we have introduced, motivated by recent observations on animal group motion, a "topological" Vicsek model in which self-propelled particles align with their Voronoi neighbors. We obtained similar results for the model in which particles interact with their $n$ (metrically) closest neighbors [15]. But this later case relies on distances and involves the additional parameter $n$, so we focused on the model presented here. At finite density, these "topological" flocking models exhibit collective properties qualitatively different from those of "metric" ones: the ordered moving phase shows novel long-range correlations (Fig. 11), even though no direct long-range connections emerge (Fig. 1fi), and the transition to collective motion exhibits critical properties different from known universality classes.

Beyond their importance in the general context of nonequilibrium order-disorder transitions, our results indicate the relevance of "topological" interactions at the collective level, following recent observations of their existence locally. Since Voronoi neighbors remain at relatively well-defined distance from each other, our approach could also provide a novel framework for modeling cohesive assemblies of cells, taking the Voronoi polygon around each particle as the effective extension of a "cell".

Nevertheless, realistic "topological" models of bird flocks, fish schools, or cell assemblies must incorporate cohesive interactions (e.g. pairwise attraction/repulsion) in order to account for the dynamics of large but finite groups in open domains. We are currently pursuing this line of research, together with theoretical investigations about the novel scaling laws uncovered here.

We acknowledge the use of the CGAL package for constructing Voronoi tesselations. Work funded in part by the French ANR projects Panurge and DyCoAct.

[1] S. Ramaswamy, Annu. Rev. Condens. Matter Phys. 1, 323 (2010).

[2] T. Vicsek, et al., Phys. Rev. Lett. 75, 1226 (1995).

[3] J.K. Parrish, S.V. Viscido, and D. Grünbaum, Biol. Bull. 202, 296 (2002), and references therein.

[4] M. Ballerini, et al., Proc. Natl. Acad. Sci. USA 105, 1232 (2008).

[5] M. Moussaïd, D.Helbing, and G. Theraulaz, "Simple heuristics govern the dynamics of human crowd motion", preprint, 2010.

[6] J. Toner, and Y. Tu, Phys. Rev. Lett.75, 4326 (1995); Phys. Rev. E 58, 4828 (1998).

[7] H. Chaté, et al., Phys. Rev. E 77, 046113 (2008); G. Grégoire and H. Chaté, Phys. Rev. Lett. 92, 025702 (2004).

[8] E. Bertin, M. Droz, and G. Grégoire, Phys. Rev. E 74, 022101 (2006); J. Phys. A 42, 445001 (2009).

[9] T. Ihle, preprint physics.bio-ph/1006.1825 (2010).

[10] M. Aldana, H. Larralde, and B. Vazquez, Int. J. Mod. Phys. B 23, 3459 (2009); G. Baglietto, and E.V. Albano, Phys. Rev. E 80, 050103 (2009).

[11] J. Toner, Y. Tu, and S. Ramaswamy, Annals of Physics 318, 170 (2005).

[12] K. Binder, Rep. Prog. Phys. 60, 487 (1997).

[13] Finite Size Scaling and Numerical Simulations of Statistical Systems, edited by V. Privman (World Scientific, Singapore, 1990); S. W. Sides, P. A. Rikvold, and M. A. 
Novotny, Phys. Rev. Lett. 81, 834 (1998).

[14] The effective $\alpha$ values recorded at fixed $L$ are larger than $\frac{8}{5}$ and converge, as $L \rightarrow \infty$, to the asymptotic value (estimated at 1.75) from below. Hence one can be sure this value is different from $\frac{8}{5}$. Note also that the metric model value $\alpha=\frac{8}{5}$ is not explicitly calculated in [6]. It can nevertheless be simply deducted from these calculations. J. Toner and S. Ramaswamy, private communication.

[15] F. Ginelli and H. Chaté, to be published. 